\title{
La promoción de la salud y la prevención de la enfermedad un reto para Terapia Ocupacional
}

Jeannette A. Méndez*

Es común escuchar las manifestaciones continuas que hacemos sobre el impacto a nivel personal y social de la realidad Colombiana. Es así como encontramos expresiones que nos reflejan miedos y temores sobre el futuro, provocando ansiedad y estrés continuo, lo cual nos deja ver la presencia de dificultades de salud mental.

La salud mental de los colombianos se ve cada vez más afectada. La violencia, los indicadores socioeconómicos negativos y la dificultad de satisfacer las necesidades son factores psicosociales que desestabilizan el equilibrio mental de las personas y por ende el desempeño de sus roles y hábitos. La tercera parte de las muertes producidas en Colombia, son resultado de un altísimo nivel de agresividad. Y las otras causales importantes de mortalidad (...) también reflejan problemas de estilos de vida y del comportamiento social.

Retomando la violencia, ésta ha sido una de las causas más importantes en el aumento de la discapacidad tanto a nivel físico como mental ya que genera el desplazamiento de familias de zonas rurales a zonás urbanas, estableciéndose en sectores marginales de la ciudad, en donde se ven enfrentados a cambios de vida, de patrones culturales, de rutinas y hábitos y la pérdida del rol laboral.

En la búsqueda de la adaptación social, la persona se ve enfrentada a cómo su saber y experiencia pueden no ser suficientes para sentirse productivos y eficaces llevando así a una pérdida del sentido de vida.

* Terapeuta Ocupacional U.N. Magíster en Desarrollo Educativo y Social. U.P.N. 
De otra parte los indicadores socioeconómicos negativos del país, ha generado aumento del desempleo, causando pérdida del rol laboral de las personas o falta de perspectivas laborales en aquellos que quieren ingresar a trabajar. En la vida cotidiana se destructuran los hábitos y rutinas conllevando a un uso inadecuado del tiempo libre, generando factores de riesgo para uso de Sustancias Psicoacttivas (SPAC) y adicciones.

Las anteriores situaciones no permiten satisfacer las necesidades del individuo, existiendo una pérdida del sentido del logro y el valor de la vida, llevando a situaciones de suicidio o intentos del mismo en jóvenes y adultos, angustia, depresión, desesperanza y aumento de factores negativos de personalidad que generan agresividad y violencia.

Socialmente encontramos expresiones de violencia intrafamiliar, social, consumo de sustancias psicoactivas, prostitución, niños y familias pobladores De y En la calle como grupos vulnerables que requieren atención y medidas de protección.

La ley 100 de Seguridad Social contempla dentro de sus postulados la equidad, el aumento de cobertura y la participación, sin embargo dentro de sus planes de beneficios en el Plan Obligatorio de Salud (POS) y el Plan Obligatorio de Salud Subsidiado (POSS), para el área de salud mental sólo cubre la consulta externa por psiquiatría o psicología y como actividades NO POS los servicios integrados de hospitalización o programas de hospital día en los cuales interviene el terapeuta ocupacional, siendo estos los servicios de rehabilitación adecuados para intervenir en procesos deteriorantes de la enfermedad mental.

En el Plan de Atención Básica (PAB) son consideradas estrategias de prevención y promoción orientadas al mejoramiento de la calidad de vida a través de estilos de vida saludables.

"Puede afirmarse, que el Sistema General de Seguridad Social en Colombia, ha sido fundamentado bajo el esquema de "Promoción y Prevención de la Enfermedad". Para asegurarlo existen poderosos mecanismos económicos, políticos y jurídicos que llevan paulatinamente el sistema hacia la búsqueda de la salud, mucho más que a la simple atención de las enfermedades"1. 
Por lo anterior, las acciones en Salud Mental deben tender a responder a la definición de Promoción de la Salud suscrita en la Carta de Otawa para la Promoción de la Salud (1986), "la cual consiste en proporcionar a los pueblos los medios necesarios para mejorar su salud y ejercer un mayor control sobre la misma. Para alcanzar un estado adecuado de bienestar físico, mental y social un individuo o grupo debe ser capaz de identificar y realizar sus aspiraciones, de satisfacer sus necesidades y de cambiar o adaptarse al medio ambiente. La salud se percibe pues, no como el objetivo, sino como la fuente de riqueza de la vida cotidiana"2.

Para complementar las acciones de promoción de la salud debe contemplarse la Prevención de la Enfermedad, la cual se considera "como un conjunto de actividades orientadas a evitar la ocurrencia de enfermedades específicas, cuyo objeto son los individuos o grupos sociales, que por sus características tengan una gran probabilidad de adquirirlas (alto riesgo)" 3 . Las estrategias de trabajo generadas tanto para la promoción y prevención deben ser desarrolladas con la población en su propio ámbito y considerando sus características y necesidades particulares.

La Asociación Americana de Terapia Ocupacinal (1989) plantea cómo la Terapia Ocupacional debe expandirse hacia actividades de promoción de la salud y prevención de la enfermedad como actividades interdependientes, las cuales deben desarrollarse en la comunidad, la escuela, la casa, el lugar de trabajo.

Así mismo, en los aportes de Eleanor Clarke (1994), propone a los terapeutas ocupacionales considerar las ideas que la comunidad tiene con respecto a la discapacidad y a la importancia del medio ambiente en la adaptación de las personas a la vida comunitaria, para establecer las estrategias de intervención que permitan el cambio y la inclusión de los pacientes a la comunidad.

Igualmente el marco de Ecología del Desempeño Humano propuesto por Down, Brown y Mc Guigan (1994) provee una estructura de pensamiento en la cual el contexto es una variable de evaluación y de planeación del tratamiento, llevando al

1 MINISTERIO DE SALUD. Promoción de la Salud y Prevención de la Enfermedad en el Sistema General de Seguridad Social en Salud. 1996.

2 OMS. Carta de Ottawa para la Promoción de la Salud. 1986.

3 MINISTERIO DE SALUD. Promoción de la Salud y Prevención de la Enfermedad en el Sistema General de Seguridad Socia en Salud. 1996. 
terapeuta ocupacional ha interesarse por la interrelación entre la persona y su contexto y el efecto de estas relaciones en su desempeño.

Estas propuestas aunque consideran el ambiente y la comunidad no han derivado en acciones de promoción y prevención primaria, sino que se han tenido en cuenta para la formulación de planes de tratamiento en casa o a nivel comuntiario. Sin embargo, la posibilidad de acción tanto en promoción como en prevención se deriva del conocimiento de los determinantes del proceso salud-enfermedad, sean estos factores genéticos, ambientales, sociales, ocupacionales, del comportamiento de los individuos o de la provisión de servicios.

En Colombia el Terapeuta Ocupacional a través de la ejecución del PAB ha empezado a desarrollar un trabajo diferente en el cual realiza en y con las comunidades sanas acciones de prevención de consumo de tabaco, alcohol y fármacos con estructurando hábitos, descarga de energía y posibilitando medio para la verbalización de las situaciones de riesgo.

Igualmente el Terapeuta Ocupacional interviene en programas de promoción de estilos de vida saludables, crecimiento y desarrollo en menores de un año, uso adecuado del tiempo libre, integración de personas con y sin discapacidad en procesos productivos, formación en Rehabilitación Basada en la Comunidad (RBC).

En programas de rehabilitación se han iniciado acciones de inclusión socioocupacional considerando los aspectos sociales y de la red de apoyo de los usuario.

Sin embargo, debemos profundizar en el estudio de la problemática que enfrentan las poblaciones vulnerables y los grupos en alto riesgo de exclusión social (desplazados por la violencia, personas con discapacidad, pobladores De y En la calle, trabajadores sexuales, farmacodependientes, madres adolescentes, grupos con violencia intrafamiliar y jóvenes con pérdida del proyecto de vida) para poder diseñar las estrategias y mecanismos protectores que propendan por su salud y por ende por su realización personal.

Realización personal que en el Modelo de Desempeño Ocupacional Realizante propone "no es un punto de llegada; se le entiende como un horizonte al que tiende la persona y al que se acerca en grados relativos, según sea la dinámica de su propio 
desempeño realizante" ${ }^{\prime \prime}$, concepto que se equipara con el ya descrito anteriormente en la Carta de Otawa.

Para lograr la integralidad y la efectividad de los programas estos deben ser el resultado de acciones intersectoriales entorno al desarrollo social de las comunidades, "la importancia de la salud, como parte de las politicas de desarrollo, depende en gran medida del grado de integración con las políticas de otros sectores, como educación, vivienda, desarrollo industrial, etc.; esta integración está sujeta al trabajo comunitario que se realice fuera del hospital"s. Es por ello que se debe participar en las Redes del Buen Trato y los Consejos Locales de Discapacidad que son las instancias que favorecen este objetivo.

Por lo anterior se hace prioritario un estudio sobre el impacto de la toma de decisiones relacionadas con las políticas de salud y las condiciones de calidad deseadas, de esta manera el Terapeuta Ocupacional debe intervenir no sólo en procesos asistenciales sino en el proceso de formulación de planes a nivel local que permitan desarrollar programas psicosociales de rehabilitación, promoción de la salud y prevención de la enfermedad con un enfoque ọcupacional.

\section{BIBLIOGRAFÍA}

AOTA. Occupational Therapy in the Promotion of Health and the Prevention of Disease and Disability. Vol. 43, No 12, 1989.

Dow. Brown, Mc Guinan. Ecology the Ocupational Behavior. ATJO. Vol 48, No 7, 1994.

Franco y Col. No violencia, salud y vida en paz. Bases para una política de enfrentamiento de la violencia desde el sector salud, 1996.

MINISTERIO DE SALUD. Promoción de la Salud y Prevención de la Enfermedad en el Sistema General de Seguridad Social en Salud, 1996.

4 TRUJILlO, Alicia. Desempeño Ocupacional Realizante; un modelo sobre los propósitos de la Terapia Ocupacional. 1995.

5 SEGOVIA, Guillermo. Bogotá hoy. Democracia, convivencia y poblaciones vulnerables. 1994. 
Reformualción de la politica colombiana en salud mental. 1996.

OMS. Carta de Otawa para la Promoción de la Salud. 1986.

SEGOVIA, Guillermo. Bogotá hoy. Democracia, convivencia y poblaciones vulnerables, 1994.

TRUJILLO, Alicia. Desempeño ocupacional realizante; un modelo sobre el propósitos de la Terapia Ocupacional. En ACTO. Vol 6, No. 2, 1995.

\section{CONGRESO LATINOAMERICANO DE BAJA VISIÓN}

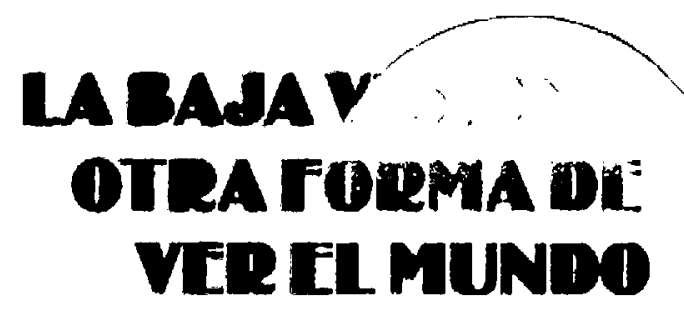

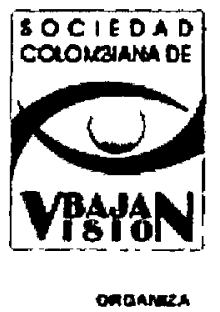

GOCKERAD COLOWIMANA of Ga da rutedin . Bebv.

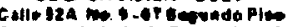

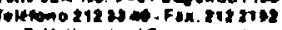

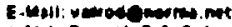

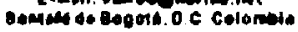

Marzo 10 y 11 - 2000

Suntafe ile Hapotí. M.C. Colentbia 